\title{
- A Case of Shell Shock
}

\section{Dharti Meshram ${ }^{1}$ Jaya Gawai' ${ }^{2}$ Pooja Kasturkar ${ }^{3}$}

\section{IJCRR}

Section: Healthcare ISI Impact Factor (2019-20): 1.628

IC Value (2019): 90.8 $\operatorname{SJIF}(2020)=7.893$

(c) (7) (8)
'MSc Nursing, DMIMSU (Deemed to be University), Smt. Radhikabai Meghe Memorial College of Nursing, Sawangi (M) Wardha, Maharashtra, India; ${ }^{2}$ Associate Professor cum HOD of MHN Dept, DMIMSU (Deemed to be University), Smt. Radhikabai Meghe Memorial College of Nursing, Sawangi (M) Wardha, Maharashtra, India; ${ }^{3}$ Assistant Professor of MHN Dept, DMIMSU (Deemed to be University), Smt. Radhikabai Meghe Memorial College of Nursing, Sawangi (M) Wardha, Maharashtra, India.

\section{ABSTRACT}

Background: Shell shock is a mental health condition caused by either suffering and observing a frightening occurrence. it involves Flashbacks, delusions or extreme nervousness, along with uncontrollable thoughts regarding the incident. Many individuals who go through traumatic experiences can have impermanent trouble adapting or managing, then they generally get well by time Respectableperson-care. shell shock symptoms get worse, for months and years, or affect through your normal working.

Goal: Raise the capacity of the old hand to make sense of stressful events or sense of normal feelings related to them.

Objective: To develop an effective manager to reduce stresses and anxiety.

Method: Knowledge used to write this case description was gathered from PubMed outlets, search hand, searching college and personal libraries looking for research techniques and case report texts, engaging in or writing many case reports with experience.

Result: The patient was received psychopharmacological treatment antidepressant drug sertraline, citalopram, fluvoxamine along with psychosocial therapy, coping strategies, family therapy, yoga, recreation, meditation and symptoms were minimized.

Conclusion: A positive outcome gets by the patient not only with the help of therapeutic management but also the family coping and support even. later on, due to proper psychopharmacological treatment, the patient showed positive feedback and slowly all the aims are achieved which were planned in the period. Finally, the patient got discharged from the hospital and now he is continuing his follow-up.

Key Words: Shell shock trauma, Neurotic disorder, Stress disorder, Flashback, Terrorism, Prolonged exposure

\section{INTRODUCTION}

Post-traumatic stress disorder (PTSD), also known as shell shock or battle fatigue syndromes, is a severe illness that may occur after an individual has encountered or witnessed a traumatic or frightening incident involving serious physical harm or danger. Shell shock is a long-term effect of traumatic experiences that trigger extreme anxiety, helplessness, or terror. ${ }^{1}$ During the Vietnam War, the idea was expressed. If it is extreme, death-threatening and reasons serious damage, an occurrence is called shocking, or the serious reaction includes fear, helplessness and terror. example of traumatic events is an accident, disaster (natural or man-made), terrorist attack, gang rape, war event, physical assault, etc. ${ }^{2}$ Most common risk factors or causes of post-traumatic stress disorder include sexual or physical assault, loss of a loved ob- ject, war, natural disaster, accident, and unexpected death of love. ${ }^{3}$ Common symptoms are relieving the traumatic events by distressing, unwanted memories, flashback, palpitation, feeling of upset, fear, feeling anger, feeling of upset, selfblame and other also, loss of interest into day to day activities, they feel cut off from friends family, want to avoid remind stressful events, they troubling to a sleeping pattern or concentration and feeling of irritable. ${ }^{4}$ Post-traumatic stress disorder developed different mental health conditions like fear, anxiety and depression, alcoholism, drugs taken as a way of coping. ${ }^{5}$ acute stress, adjustment disorder, disinhibited social engagement disorder, etc. The post-traumatic stress disorder prevalence rate among adults is $6.8 \%$. the lifetime prevalence rate among men was 3.6 and in females was $9.7 \%{ }^{6}$

\section{Corresponding Author:}

Dharti Meshram, MSc Nursing, DMIMSU (Deemed to be University), Smt. Radhikabai Meghe Memorial College of Nursing, Sawangi (M) Wardha, Maharashtra, India; Email: dhartimeshram9@gmail.com

ISSN: 2231-2196 (Print)

Received: 12.01 .2021
ISSN: 0975-5241 (Online)

Revised: 22.03 .2021
Accepted: 24.07.2021
Published: 01.12 .2021 
The COVID-19 developed various kinds of post-traumatic stress disorder such as anxiety or, stress, depressive disorder, suicide, substance use disorders, loss of job, income, failure of a marriage, etc. Global effects include high morbidity or mortality rate or financial problem, sustained social isolation for people. Isolation, quarantine, and lockdown measures to control the COVID-19 may have negative and psychological, and social consequences. The majority of the expected direct effects of quarantine and related social and physical isolation, such as financial insecurity, boredom, anger, feeling burdened, loneliness, and fear, are risk factors for mental health problems such as anxiety, depression, suicide, and self-harm. ${ }^{7}$

\section{Patient Information}

A case selected from AVBR Hospital Sawangi (Meghe), Wardha where lack of mental health services for the remote population, or Acharya Vinoba Bhave Rural Hospital provided psychiatric health facilities for all the needy people.

The 35-year-old woman had a history of losing her son in a road traffic accident three months ago. she detached herself from social, lack interaction with others, from a family member, friends. she was talking about the incident continuously for 3 week, irritable, muttering to herself, loss of interest in daily activity, disturbed sleeping pattern, anxious, worried, stressed, loss of appetites, hallucination present, and muttering to self. No history of the family regarding mental illness. all necessary investigations are done like history collection, mental status examination, blood investigation after that diagnosed to have post-traumatic stress disorder by mental status examination.

Precipitating factors: stressor, the presence of a traumatic event with her son.

\section{Past psychiatric history}

The patient was asymptomatic 3 months before. Due to the loss of their love object (son), she has started symptoms she loss of social interaction with others, irritability, muttering to self, loss of interest in daily activity, disturbed sleeping pattern, anxiety, worried, stress, loss of appetites, muttering to self, hallucination. the patient was taken treatment from a private hospital. he received psychopharmacological treatment antidepressant, fluoxetine, mood stabilized. still symptoms not minimized. so, a patient came to AVBR Hospital Sawangi Meghe Wardha.

The patient is not having any past and present medical history apart from this. The patient was completely alright before the presentation of the above clinical manifestations. No history of surgery.

Family history: patient belongs to a joint family, and is middle class. her life with her husband and daughter and moth- er-in-law and father-in-law. her husband working as clear in private, his family income per month $18000 /-$. she is a housewife.

\section{Sign and symptoms}

This patient presented with typical symptoms of loss of social interaction with others, irritability, muttering to self, loss of interest in daily activity, disturbed sleeping pattern, anxiety, worried, stress, loss of appetites, muttering to self, hallucination. ${ }^{8}$

\section{Diagnostic Assessment}

History collection done no any history of the family regarding mental illness.

Physical examination: Facial expression tension, anxiety, unstable gait, lack of speech.

Blood investigation: $\mathrm{Hb} \%-11 \mathrm{mg} / \mathrm{dl}$, glucose plasma random-80 mg\%, serum globulin $3.0 \mathrm{gm} \%$, serum protein$7 \mathrm{gm} \%$, and antigen test- Negative.

Mental status examination: auditory hallucination present, abnormal speech, abnormal, inappropriate affect. Neurological examination and process recording are done.

\section{Therapeutic intervention.}

Fluoxetine/Prozac-By blocking the reuptake transporter protein located in the presynaptic terminal, it helps block the reuptake of serotine into presynaptic neurons.

Sertraline/Zoloft-As an inhibitor of dopamine transporters, it has a mild effect.

Imipramine/Tofranil-It helps to inhibit the reuptake of certain neurotransmitters in the brain.

Nursing interventions - provided supportive therapy to develop trust, reduce the stress and anxiety of the client. Avoid excessive probing into details of trauma Promote independence and the client's higher level of functioning. Reconnect the individual with the existing support system explained stress coping strategies therapy yoga, meditation, relaxation, recreation, group therapies to reduce isolation, breathing techniques. A consistent empathetic approach helps the client to tolerate intense memories and emotional pains.

\section{Timeline}

The patient was admitted for three weeks and the nursing care has been provided along with the psychopharmacological interventions along with psychosocial therapy like mediation, yoga recreation therapy, stress management coping strategies, Cognitive Behaviour Therapy, group, and family therapy. The patient's condition was prognosed and the patient got discharged from the hospital in a good condition and follow-up has been taken regularly. 
Data extraction: Data collected from PubMed, libraries hand search book, Medline and Cochrane.

\section{Primary Outcome}

To minimize symptoms of shell shock by the antianxiety drug, mood stabilizer antidepressant drug and other psychosocial therapies.

\section{Secondary outcome}

Regular follow-up will prevent adverse effects of newly prescribed antipsychotic drugs.

\section{Prognosis}

The prognosis is the first and foremost dependant on early and successful treatment of post-traumatic stress disorder. As well as the prognosis also depends upon the severity of the disease condition but also the socioeconomic background of the family and meanwhile family coping. treatment regimen, later on, the patient was given a positive outcome and finally, he was discharged from the hospital while staying in a hospital for about 20 days.

\section{DISCUSSION}

A 35 year of old female client from Nalwadi was admitted to AVBR Hospital Sawangi Meghe Wardha in a female psychiatric ward with chief complaints of muttering to self, hallucination, lack of social interaction with a family member and others, anxiety, tension, stress, repetition of events, flash block for 3 weeks caused by this her lose her son in a road traffic accident. the patient received psychopharmacological treatment along with psychosocial therapy. only pharmacological treatment is not effective but psychosocial therapy is also important for reducing symptoms. The patient's condition was improved and finally, the patient got discharged from the hospital and she has taken follow-up services in Acharya Vinoba Bhave Rural Hospital on an OPD basis.

One of the investigators found that approximately 3.6 per cent of American adults, or 5.2 million individuals, have experience shell shock in a given year, and found that 7.8 million Americans will develop shell shock at some stage in their lives. It occurs in any age group, including childhood. Most common in women rather than men due to women being more likely to be victims of domestic violence, bullying, and rape. ${ }^{2}$

One of the researchers researched Evidence-based post-traumatic stress disorder trials for the third generation. Related EB clinical trials are expected in due course to assess the intensity of the indication in support of alternative methods to the management of shell shock. Like that the practice of complementary treatments (e.g., Herbal massage and food complements) for tension or co-morbid pain, nervousness and fear are common between active military veterans or their partners. Results suggest that despite sound positive research information, around seventy per cent of the surveyed subjects want these treatments existing at the medical management services. Thus, this patient group is at significant risk of being underserved. The need for orderly evaluations of the benefit of complementary medicine in the action of posttraumatic stress disorder between the civilian people is also becoming apparent in the light of growing studies suggesting the profits of acupuncture or massage in people exposed to traumatic events. ${ }^{9}$

One of the research articles says that Rates of Post-Traumatic Stress Disorder were observed in instruction to comparison the outline inpatient of an Australian Community Mental Health Facility through that was described in the international literature for clients with a major mental disorder or to discover the outcome of this on client health outcomes. they used t-test and ANOVA. in that finding of the result is undocumented trauma and post-traumatic stress disorder were found. It is assumed that, as stated for people with a serious mental condition, the elevated stages of the event, with those observed in the present research, are primarily linked to the features of the people receiving confirm community mental health facilities or that these factors have exact consequences for the distribution of facilities or increase problems of source utilization or efficiency. ${ }^{10}$

One of the researches conducted on trauma-related health problem humanitarian staff. The result of this study suggests that mental health issues are faced by nationwide workers or the prevalence of PTSD, anxiety or depression, in this occupation category is generally equal to or greater than amongst comparison groups. There is an especially scarce study amongst nationwide workers on substance use illness or suicidal behaviour. The relationship between gender or mental health problems amongst nationwide workers seems to be complex, or support for organizational staff seems to be a significant factor of mental health. ${ }^{11}$

\section{CONCLUSION}

Posttraumatic stress disorders are psychiatric illnesses with substantial psychosocial and biological consequences. These disorders can cause significant emotional and physical turmoil. In delivering appropriate treatment for the client, nurses can involve members of a family or build support and a clear empathic method to help the client withstand penetrating memories or emotional pain. Posttraumatic stress disorder, on the other hand, provides an opportunity for psychological and spiritual development due to the human capacity to adapt or succeed in the face of hardship or difficult times. 


\section{ACKNOWLEDGEMENT}

The author special thanks to Mrs Jaya Gawai associate professor cum HOD of the mental health department for supporting and guiding from time to time. also, thanks to Mrs Pooja Kasturkar assistance professor of mental health departments of nursing Smt. Radhikabai Meghe memorial college of nursing. Datta Meghe institution of medical sciences (Deemed to be University) Sawangi Meghe Wardha Maharashtra, India for suggestion and guidance.

\section{Author contribution}

All author equally participates in this case report.

Informed Consent: Written Inform consent of the patient was taken before select the case.

Interest and conflict: Nil

Financial resource of the study: Nil

\section{REFERENCES}

1. Jones E, Wessely S. A paradigm shift in the conceptualization of psychological trauma in the 20th century. Journal of anxiety disorders. 2007 Jan 1;21(2):164-75.

2. O'Connell WE, Hooker E. Anxiety disorders II. Psychopathology \& psychotherapy: From DSM-IV diagnosis to treatment. 1996:179-86.

3. Newman E, Simpson R, Handschuh D. Trauma exposure and post-traumatic stress disorder among photojournalists. Visual Communication Quarterly. 2003 Jan 1;10(1):4-13.
4. Briere JN, Scott C. Principles of trauma therapy: A guide to symptoms, evaluation, and treatment (DSM-5 update). Sage Publications; 2014 Mar 25.

5. Batten SV, Hayes SC. Acceptance and commitment therapy in the treatment of comorbid substance abuse and post-traumatic stress disorder: A case study. Clinical case studies. 2005 Jul;4(3):246-62.

6. Friedman MJ. Posttraumatic and acute stress disorders. Springer; 2015 Feb 28.

7. Wang Y, Shi L, Que J, Lu Q, Liu L, Lu Z, Xu Y, Liu J, Sun Y, Meng S, Yuan K. The impact of quarantine on mental health status among general population in China during the COVID-19 pandemic. Molecular psychiatry. 2021 Jan 22:1-0.

8. Brown TA, Barlow DH, DiNardo PA. Anxiety disorders interview schedule adult version: Client interview schedule. Grey wind Publications Incorporated; 1994.

9. Batelaan NM, Bosman RC, Muntingh A, Scholten WD, Huijbregts KM, van Balkom AJ. Risk of relapse after antidepressant discontinuation in anxiety disorders, obsessive-compulsive disorder, and post-traumatic stress disorder: systematic review and meta-analysis of relapse prevention trials. BMJ. 2017 Sep $13 ; 358$.

10. Friedman MJ, Schnurr PP, McDonagh-Coyle A. Post-traumatic stress disorder in the military veteran. Psychiatric Clinics. 1994 Jun 1;17(2):265-77.

11. Xiong T, Wozney L, Olthuis J, Swati Singh R, McGrath P. A scoping review of the role and training of paraprofessionals delivering psychological interventions for adults with post-traumatic stress. J Dep Anxiety. 2019;8(342):2167-1044. 